\title{
NDT-Tool: A Case Tool to Deal with Requirements in Web Information Systems
}

\author{
M.J. Escalona, J. Torres, M. Mejías, and A. Reina \\ Department of Computer Languages and Systems \\ University of Seville \\ \{escalona, jtorres, risoto, reinaqu\}@lsi.us.es
}

\begin{abstract}
Internet progress and the rising interest for developing systems in web environment has given way to several methodological proposals which have been proposed to be a suitable reference in the development process. However, there is a gap in case tool[3][4][6]. This work presents a case tool named NDT-Tool that allows to apply algorithms and techniques proposed in NDT (Navigational Development Techniques) [2], which is a methodological proposition to specify, analyze and design web information systems.
\end{abstract}

\section{Introduction}

In the last years, several methodological proposals have been developed by the research community: OOHDM[7], UWE[5], WebML[1] or, UWA project[8] are only some examples. However, after studying them and analyzing several comparative studies [4][6], we can deduce most of them are mainly focused on the design and implementation phases. They often do not offer a guide to deal with web requirements and, they often propose use cases and only some of them, like UWE, propose techniques to describe or validate requirements. This paper offers a global vision of NDT, a web methodological proposal that is focused on the first phases of the life cycle and it presents a case tool, NDT-Tool, which guides in NDT application.

\section{NDT (Navigational Development Technique)}

The first phase in NDT, requirements treatment, starts capturing requirements and studying the environment. NDT proposes to use some requirements capture techniques like interviews and brainstorming. After this phase, the objectives must be captured and described. The process continues based on these objectives and proposes to define the system requirements. NDT classifies requirements into storage information, actor, functional, interaction and non-functional requirements. Each kind of them is described using a pattern. After that, and starting with these patterns three design models can be obtained systematically: the conceptual model, that expresses the static aspect of the system; the navigational model, that describes how the user can navigate in the system; and the abstract interface model, that expresses interface.

\section{NDT-Tool}

NDT-Tool is a case tool, which allows to apply NDT algorithms and techniques and to generate results, documents and models automatically. Nowadays, only require- 
ments phase is completely codified, but some algorithms and models of the next phases are already implemented. The first form in NDT offers a global menu with several options: management project, requirements treatment, conceptual model, navigational model, interface model and prototypes generation option. Models in the conceptual model, the navigational model and the abstract interface model can be obtained automatically and visualized in Rational Rose environment. Nowadays, algorithms which generate these models are implemented but we are still working in the connection with the Rational Rose tool.

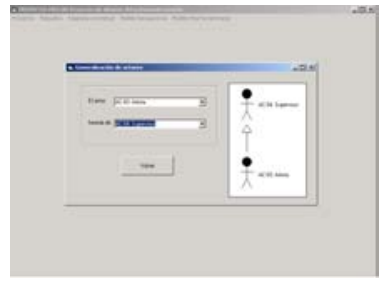

a

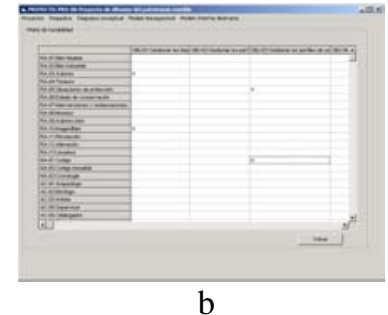

Fig. 1. Some screens of NDT

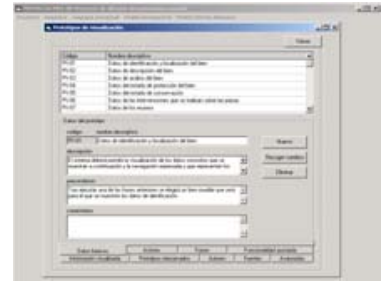

$\mathrm{c}$

In the figure 1, some screens of NDT are shown. In a, the graphical option to work with actors and roles in requirements definition is offered. In $b$, the matrix of trazability which makes possible to evaluate if all the objectives have been covered in the specification is presented and in c, a example of a requirements pattern is shown. Data in this screen has been obtained from a real system developed with NDT and NDT-Tool. NDT has been applied to several real problems giving very good results. Now, we are applying our proposal of NDT-Tool to these examples to evaluated it.

\section{References}

1 Ceri, S. Fraternai, P., Bongio, A. Web Modelling Language (WebML): A Modelling Language for Designing Web Sites. Proc. of WWW9/Computer Networks 332000

2 Escalona, M.J.,Mejías, M., Torres, J. Reina.A. NDT: Una técnica para el desarrollo de la navegación. Congreso IDEAS 2002. Cuba. Abril 2002

3 Escalona, M.J., Koch, N. Ingeniería de Requisitos en Aplicaciones para la Web-Un estudio comparativo. Technical report. Dep. Language and Computer Science. Seville. 2002.

4 Koch, N. A comparative study of methods for Hypermedia Development. Technical Report 9905. Ludwig-Maximilian-University, Munich, Germany. 1999.

5 Koch, N. Software Engineering for Adaptative Hypermedia Applications. Ph. Thesis, FAST Reihe Softwaretechnik Vol(12), Uni-Druck Publishing Company, Munich. Germany. 2001.

6 Retschitzegger, W. \& Schwinger, W. Towards Modeling of Data Web Applications - A Requirement's Perspective. American Conference on Informatin Systems AMCIS 2000,

7 G. Rossi An Object Oriented Method for Designing Hipermedia Applications. PHD Thesis, Departamento de Informática, PUC-Rio, Brazil, 1996

8 UWA Consortium, UWA Requirements Elicitation: Model, Notation, and Tool Architecture. 